\title{
L-Carnitine preserves endothelial function in a lamb model of increased pulmonary blood flow
}

\author{
Shruti Sharma ${ }^{1}$, Angela Aramburo ${ }^{2,3}$, Ruslan Rafikov ${ }^{1}$, Xutong Sun', Sanjiv Kumar' ${ }^{1}$, Peter E. Oishii, ${ }^{2,4}$, Sanjeev A. Datar ${ }^{2}$, Gary Raff $^{5}$,
} Kon Xoinis ${ }^{2}$, Gohkan Kalkan'2 , Sohrab Fratz ${ }^{6}$, Jeffrey R. Fineman ${ }^{2,4}$ and Stephen M. Black ${ }^{1}$

BACKGROUND: In our model of a congenital heart defect (CHD) with increased pulmonary blood flow (PBF; shunt), we have recently shown a disruption in carnitine homeostasis, associated with mitochondrial dysfunction and decreased endothelial nitric oxide synthase (eNOS)/heat shock protein (Hsp)90 interactions that contribute to eNOS uncoupling, increased superoxide levels, and decreased bioavailable nitric oxide (NO). Therefore, we undertook this study to test the hypothesis that L-carnitine therapy would maintain mitochondrial function and NO signaling.

METHODS: Thirteen fetal lambs underwent in utero placement of an aortopulmonary graft. Immediately after delivery, lambs received daily treatment with oral L-carnitine or its vehicle.

RESULTS: L-Carnitine-treated lambs had decreased levels of acylcarnitine and a reduced acylcarnitine:free carnitine ratio as compared with vehicle-treated shunt lambs. These changes correlated with increased carnitine acetyl transferase (CrAT) protein and enzyme activity and decreased levels of nitrated CrAT. The lactate:pyruvate ratio was also decreased in L-carnitine-treated lambs. Hsp70 protein levels were significantly decreased, and this correlated with increases in eNOS/Hsp90 interactions, NOS activity, and $\mathrm{NO}_{x}$ levels, and a significant decrease in eNOS-derived superoxide. Furthermore, acetylcholine significantly decreased left pulmonary vascular resistance only in L-carnitine-treated lambs.

CONCLUSION: L-Carnitine therapy may improve the endothelial dysfunction noted in children with CHDs and has important clinical implications that warrant further investigation.

hildren with congenital heart defects (CHDs) that result in increased pulmonary blood flow (PBF) develop early and progressive alterations in pulmonary vascular function that cause significant morbidity (1). The mechanisms involved in this pulmonary vascular disease are not fully understood; however, secondary endothelial injury is thought to be an early hallmark. The most important consequence of endothelial injury is a decrease in bioavailable nitric oxide (NO), with subsequent endothelial dysfunction, or impaired ability of the endothelium to mediate vasodilation (2). Compelling evidence suggests that impaired NO signaling and oxidative stress play a key role in these events (3).

Oxidative stress occurs when generation of reactive oxygen species (ROS) overwhelms the cells' natural antioxidant defenses, resulting in cellular damage and impaired function of vulnerable tissues. Four enzyme systems are thought to predominate in vascular endothelial ROS generation: nicotinamide adenine dinucleotide phosphate oxidase, xanthine oxidase, uncoupled endothelial nitric oxide synthase (eNOS), and mitochondrial electron leakage. Whereas the former three have been extensively studied, the role of mitochondrial-derived ROS in the vascular endothelium has received less attention (4). Mitochondria, through oxidative phosphorylation, are considered the major source of ROS in most mammalian cells. At the same time, mitochondria are potential targets of ROS action. Therefore, increased ROS can damage DNA, proteins, and lipids within the mitochondria, leading to alterations in the respiratory chain resulting in decreased energy production and a further increase in ROS generation ("ROS-induced ROS release") $(5,6)$. In recent years, it has become clear that mitochondrial dysfunction is a critical event in numerous pathologic conditions associated with oxidative stress, including diabetes mellitus, chronic renal failure, and neurodegenerative or cardiovascular diseases (4,7-9). The contribution of the mitochondria, however, to the pathogenesis of pulmonary vascular disease remains poorly understood.

Previously, we have established a clinically relevant animal model of a CHD with increased PBF, by placing a large aortopulmonary vascular graft (shunt) into the late-gestation fetal lamb (10). This allows the study of early mechanisms of pulmonary vascular disease. In this model, we have shown a selective impairment of endothelium-mediated pulmonary vasodilation (11), associated with decreased NO signaling and increased oxidative stress $(3,12,13)$. Recently,

The first two authors contributed equally to this work.

'Pulmonary Vascular Disease Program, Vascular Biology Center, Georgia Regents University, Augusta, Georgia; ${ }^{2}$ Department of Pediatrics, University of California,

San Francisco, San Francisco, California; ${ }^{3}$ Department of Pediatrics, Autonomous University of Barcelona, Barcelona, Spain; ${ }^{4}$ Cardiovascular Research Institute, University of California, San Francisco, San Francisco, California; ${ }^{5}$ Department of Cardiothoracic Surgery, University of California, Davis, Davis, California; ${ }^{6}$ Department of Pediatric Cardiology and Congenital Heart Disease, Deutsches Herzzentrum München, Klinik an der Technischen Universität München, Munich, Germany. Correspondence: Stephen M. Black (SBLACK@georgiahealth.edu) 


\section{Articles | Sharmaet al.}

we also demonstrated a disruption in carnitine homeostasis in shunt lambs, correlated with mitochondrial dysfunction and decreased eNOS/Hsp90 interactions, which contributed to eNOS uncoupling and decreased NO signaling (14). Carnitine plays an important role in cellular energy metabolism and is essential for mitochondrial health (15). However, whether carnitine supplementation can modify the course of pulmonary vascular disease secondary to increased PBF is unknown. Therefore, the purpose of this study was to determine if chronic supplementation with L-carnitine would

Table 1. Baseline hemodynamic variables and blood gases

\begin{tabular}{lccc}
\hline Hemodynamic variable & $\begin{array}{c}\text { Control } \\
(n=5)\end{array}$ & $\begin{array}{c}\text { Shunt } \\
(n=6)\end{array}$ & $\begin{array}{c}\text { Shunt }+ \\
\text { carnitine } \\
(n=6)\end{array}$ \\
\hline mPAP $(\mathrm{mm} \mathrm{Hg})$ & $15.3 \pm 3.8$ & $23.1 \pm 4.3^{*}$ & $21.2 \pm 3.6$ \\
$\mathrm{mSAP}(\mathrm{mm} \mathrm{Hg})$ & $77.6 \pm 7.7$ & $70.7 \pm 8.9$ & $60.0 \pm 6.8^{*}$ \\
$\mathrm{mRAP}(\mathrm{mm} \mathrm{Hg})$ & $3.5 \pm 2$ & $3.8 \pm 1.4$ & $4.2 \pm 1.3$ \\
$\mathrm{mLAP}(\mathrm{mm} \mathrm{Hg})$ & $4.1 \pm 1.6$ & $7.1 \pm 1.7$ & $9.0 \pm 3.1^{*}$ \\
$\mathrm{HR}$ & $126 \pm 13$ & $174 \pm 26^{*}$ & $144 \pm 40$ \\
$\mathrm{Q}_{\mathrm{lpa}}(\mathrm{ml} / \mathrm{min} / \mathrm{kg})$ & $0.8 \pm 0.2$ & $1.8 \pm 0.6$ & $2.1 \pm 0.8^{*}$ \\
$\mathrm{LPVR}(\mathrm{mm} \mathrm{Hg} / \mathrm{ml} / \mathrm{min} / \mathrm{kg})$ & $0.24 \pm 0.10$ & $0.14 \pm 0.06$ & $0.08 \pm 0.02^{*}$ \\
$\mathrm{Qp}: \mathrm{Qs}$ & & $2.8 \pm 0.9$ & $2.8 \pm 0.4$ \\
$\mathrm{pH}$ (units) & $7.36 \pm 0.03$ & $7.40 \pm 0.02$ & $7.41 \pm 0.02$ \\
$\mathrm{pCO}_{2}$ (torr) & $42.3 \pm 2.1$ & $40.0 \pm 3.7$ & $42.0 \pm 2.0$ \\
$\mathrm{pO}_{2}$ (torr) & $78.3 \pm 12.2$ & $70.7 \pm 11.2$ & $75.4 \pm 14.3$ \\
$\mathrm{Hb}_{\mathrm{H}}$ concentration & $9.0 \pm 1.7$ & $9.8 \pm 1.6$ & $8.7 \pm 1.1$ \\
Weight (kg) & $15.7 \pm 2.5$ & $13.9 \pm 2.1$ & $13.4 \pm 2.7$ \\
\hline
\end{tabular}

Values are mean \pm SD.

$\mathrm{Hb}$, hemoglobin; HR, heart rate; LPVR, left pulmonary vascular resistance; $\mathrm{mLAP}$, mean left atrial pressure; mPAP, mean pulmonary arterial pressure; mRAP, mean right atrial pressure; $m S A P$, mean systemic arterial pressure; $\mathrm{Q}_{1 \mathrm{pa}^{\prime}}$, blood flow through the left pulmonary artery; Qp:Qs, ratio of pulmonary to systemic blood flow.

${ }^{*} P<0.05$ control vs. vehicle-treated shunt. ${ }^{*} P<0.05$ control vs. shunt + carnitine. attenuate oxidative stress and preserve carnitine homeostasis, mitochondrial homeostasis, and $\mathrm{NO}$ signaling in our lamb model of CHD with increased PBF and thereby result in improved endothelial function.

\section{RESULTS}

\section{Hemodynamics}

The baseline hemodynamic data, hemoglobin concentration, and systemic arterial blood gases for carnitine-treated shunt lambs, vehicle-treated shunt lambs, and age-matched control lambs at 4 wk of age are shown in Table 1. There were no statistically significant differences in baseline hemodynamic indexes between the two shunt groups. There were also no differences in hemoglobin concentrations, arterial blood gases, or ventilator parameters among the three groups. The shunt fraction (Qp/Qs) was 2.8 in each group, demonstrating the large aortopulmonary shunt. However, as compared with age-matched control lambs, the significant increases in mean pulmonary arterial pressure and heart rates in vehicle-treated shunt lambs was not observed in carnitine-treated shunt lambs. Carnitine treatment also decreased mean systemic arterial pressure and left pulmonary vascular resistance as compared with agematched control lambs. Blood flow through the left pulmonary artery and mean left atrial pressure were also significantly increased in carnitine-treated shunt lambs as compared with age-matched controls.

\section{Evaluation of the Proteins Responsible for Maintaining Carnitine Homeostasis}

As compared with vehicle-treated shunt lambs, carnitinetreated shunt lambs displayed an increase in the peripheral lung protein levels of carnitine acetyl transferase (CrAT) (Figure 1a). Furthermore, CrAT activity was significantly higher in carnitine-treated shunt lambs as compared with vehicle-treated shunt lambs (Figure 1b). The level of nitrated
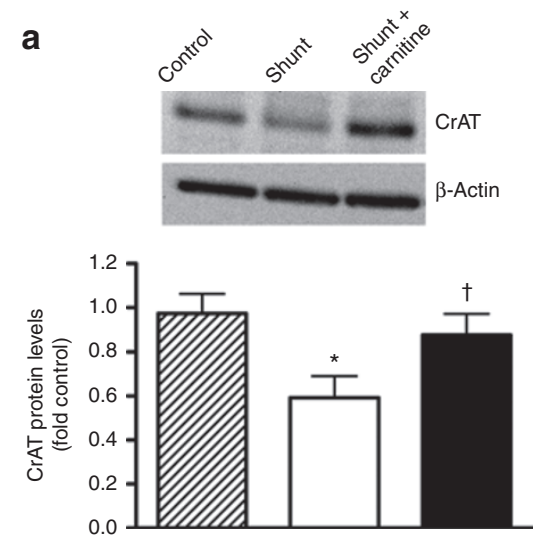

b

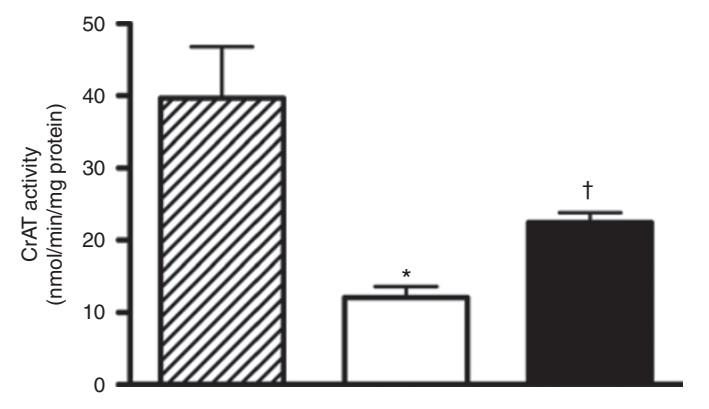

C

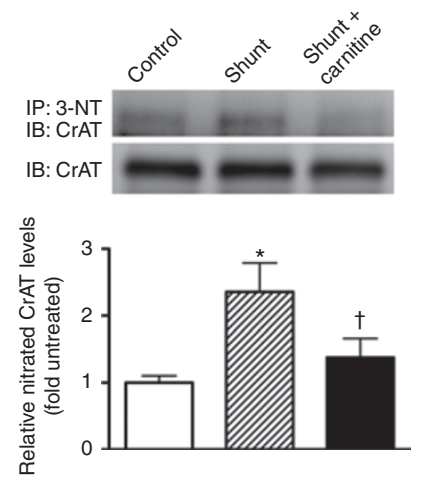

Figure 1. Carnitine acetyltransferase (CrAT) protein levels and activity in the lamb lung. (a) Protein extracts $(50 \mu \mathrm{g})$, prepared from peripheral lung of vehicle- (white) and carnitine-treated (black) shunt lambs as well as age-matched control lambs (hatched) were analyzed by western blot analysis using a specific antiserum raised against CrAT protein. Blots were also normalized for loading using $\beta$-actin. A representative blot is shown. (b) CrAT activity was determined in protein extracts $(40 \mu \mathrm{g})$ prepared from peripheral lung tissue from all three groups of lambs. (c) Protein extracts (1 mg) were also subjected to immunoprecipitation using an antibody specific to 3-NT and then analyzed by western blot analysis using a specific antiserum raised against CrAT protein. A representative blot is shown. Values are mean $\pm \mathrm{SEM} ; n=6$ vehicle-treated shunt lambs, $n=7$ carnitine-treated shunt lambs, and $n=4$ agematched control lambs. ${ }^{*} P<0.05$ vs. control; ${ }^{\dagger} P<0.05$ vs. vehicle-treated shunt lambs. IB, immunoblot; IP, immunoprecipitation. 
a

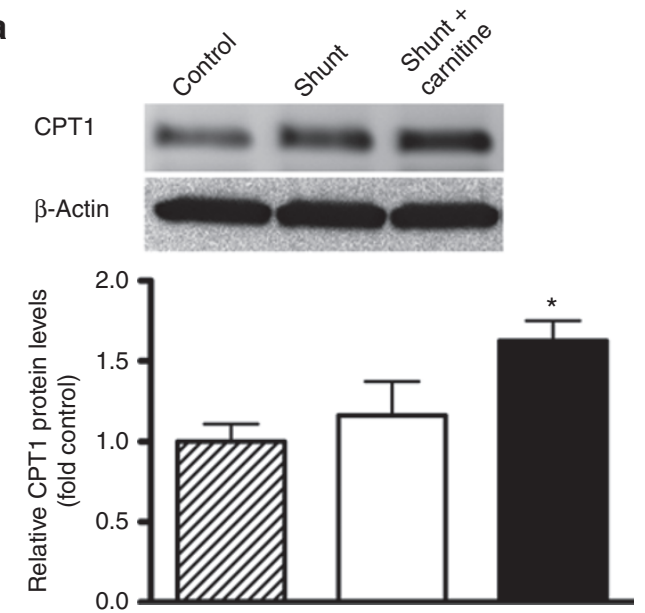

b
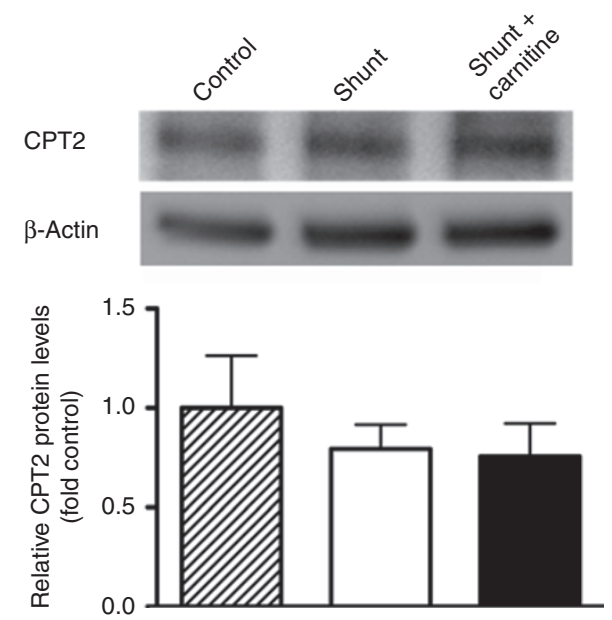

Figure 2. Carnitine palmitoyltransferase $1 B(C P T 1)$ and carnitine palmitoyltransferase 2 (CPT2) protein levels in the lamb lung. (a,b) Protein extracts $(50 \mu \mathrm{g})$, prepared from peripheral lung of vehicle- (white) and carnitine-treated (black) shunt lambs as well as age-matched control lambs (hatched) were analyzed by western blot analysis using specific antisera raised against CPT1 and CPT2. Blots were also normalized for loading using $\beta$-actin. Representative blots are shown. Values are mean $\pm \mathrm{SEM} ; n=6$ vehicle-treated shunt lambs, $n=7$ carnitine-treated shunt lambs, and $n=5$ age-matched control lambs. ${ }^{*} P<0.05$ vs. control.

CrAT was also significantly reduced in carnitine-treated shunt lambs as compared with vehicle-treated shunt lambs (Figure 1c). Furthermore, although CrAT protein levels (Figure 1a) and nitrated CrAT (Figure 1c) were not significantly different from those of age-matched control lambs with normal PBF, CrAT activity was still significantly reduced (Figure $\mathbf{1 b}$ ). Furthermore, the peripheral lung protein levels of carnitine palmitoyltransferase 1 (CPT1) was significantly higher in carnitine-treated shunt lambs as compared with age-matched control lambs with normal PBF (Figure 2a), whereas CPT2 levels were unchanged in all three groups (Figure 2b).

\section{Evaluation of Carnitine Homeostasis}

In the current study, we determined peripheral lung carnitine levels in carnitine- and vehicle-treated shunt lambs as well as age-matched control lambs with normal PBF. We found that acyl carnitine levels were significantly higher in vehicle-treated

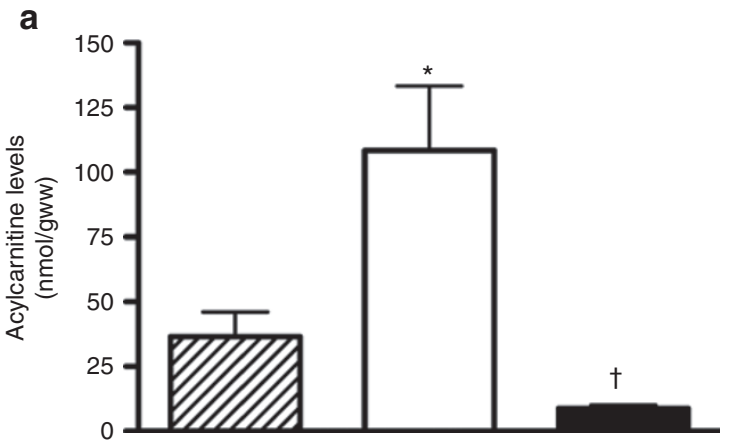

b

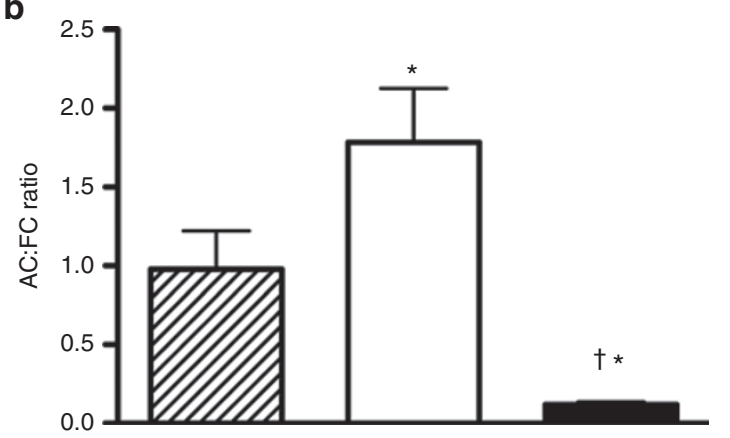

Figure 3. Carnitine homeostasis in the lamb lung. (a) Acylcarnitines and (b) the acylcarnitine:free carnitine (AC:FC) ratio were determined in peripheral lung of vehicle- (white) and carnitine-treated (black) shunt lambs as well as age-matched control lambs (hatched). Values are mean $\pm \mathrm{SEM} ; n=6$ vehicle-treated shunt lambs, $n=7$ carnitine-treated shunt lambs, and $n=4$ age-matched control lambs. ${ }^{*} P<0.05$ vs. control; ${ }^{\dagger} P<$ 0.05 vs. vehicle-treated shunt lambs.

shunt lambs than in either carnitine-treated shunt lambs or age-matched control lambs with normal PBF (Figure 3a). Similarly, the peripheral lung acylcarnitine:free carnitine ratio was significantly higher in vehicle-treated shunt lambs than in either carnitine-treated shunt lambs or age-matched control lambs with normal PBF (Figure 3b). In addition, although the acylcarnitine levels were unchanged between carnitine-treated shunt lambs and age-matched control lambs with normal PBF, the acylcarnitine:free carnitine ratio was significantly lower (Figure 3b).

\section{Evaluation of Mitochondrial Function}

In the current study, we determined and compared the pulmonary levels of lactate and pyruvate to estimate lung mitochondrial activity. As shown in Figure 4a, the lactate:pyruvate ratio was significantly higher in vehicle-treated shunt lambs than in either carnitine-treated shunt lambs or age-matched control lambs with normal PBF (Figure 4a). The lactate:pyruvate ratio was unchanged in age-matched control lambs with normal PBF as compared with shunt lambs treated with L-carnitine (Figure 4a). In addition, our data indicate that Hsp70 protein levels were significantly higher in vehicle-treated shunt lambs than in either carnitine-treated shunt lambs or age-matched control lambs with normal PBF (Figure 4b). The levels of Hsp70 were not different between carnitine-treated shunt lambs and age-matched control lambs with normal PBF (Figure 4b). 
a

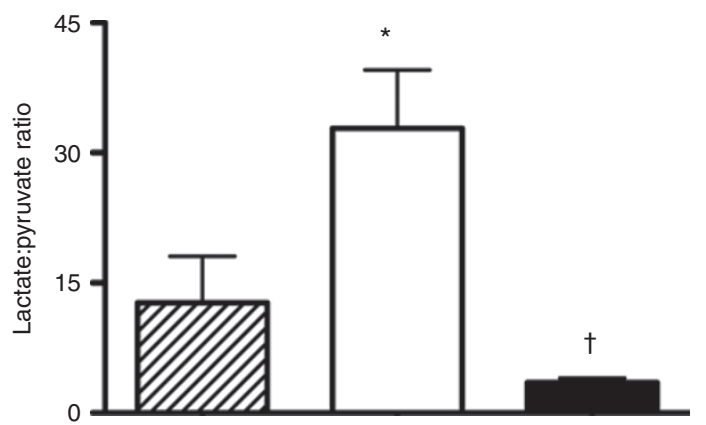

b

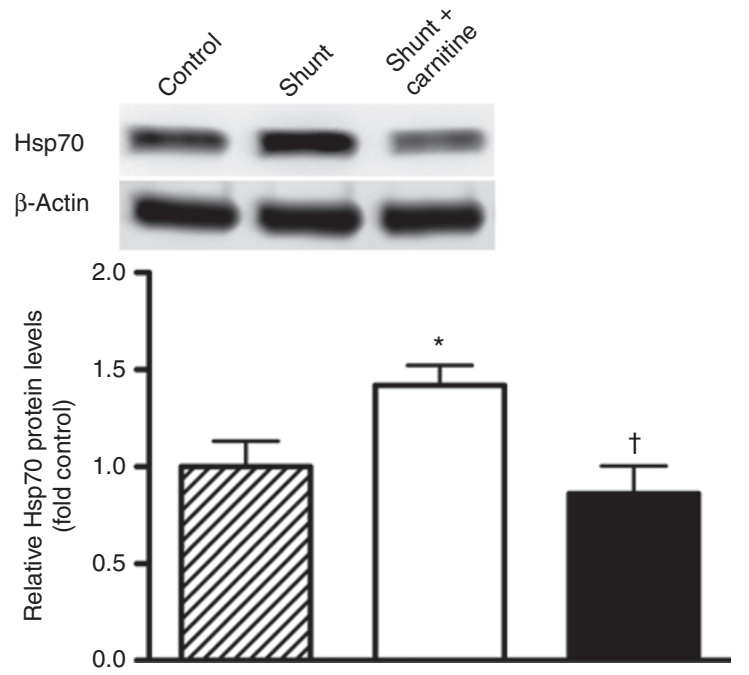

Figure 4. Hsp90-eNOS interactions in the lamb lung. (a) The lactate:pyruvate ratio was determined in peripheral lung of vehicle (white)- and carnitine (black)-treated shunt lambs as well as age-matched control lambs (hatched). (b) Protein extracts $(50 \mu \mathrm{g})$, prepared from all three groups were also analyzed by western blot analysis using a specific antisera raised against $\mathrm{Hsp} 70$. Blots were also normalized for loading using $\beta$-actin. A representative blot is shown. Values are mean $\pm S E M ; n=6$ vehicle-treated shunt lambs, $n=7$ carnitine-treated shunt lambs, and $n=$ 4 age-matched control lambs. ${ }^{*} P<0.05$ vs. control; ${ }^{+} P<0.05$ vs. vehicletreated shunt lambs. eNOS, endothelial nitric oxide synthase; Hsp, heat shock protein.

Determinations of eNOS-Hsp90 Interactions, Superoxide Levels, and NO Signaling

In the current study, we determined and compared these parameters in carnitine and vehicle-treated shunt lambs as well as age-matched control lambs with normal PBF. We found that eNOS-bound Hsp90 was significantly lower in vehicletreated shunt lambs as compared with either carnitine-treated shunt lambs or age-matched control lambs with normal PBF (Figure 5a). Hsp90-eNOS interactions were not significantly different between carnitine-treated shunt lambs and agematched control lambs with normal PBF (Figure 5a). In addition, we found a decrease in NOS-dependent superoxide levels, indicative of decreased eNOS uncoupling, in carnitine-treated shunt lambs as compared with vehicle-treated shunt lambs (Figure 5b). Again, NOS-derived superoxide levels were not significantly different between carnitine-treated shunt lambs and age-matched control lambs with normal PBF (Figure 5b). The maximum rate of the reaction for total NOS activity in the peripheral lung was also significantly higher in carnitinetreated shunt lambs as compared with both vehicle-treated shunt lambs and age-matched control lambs with normal PBF (Figure 5c). Finally, the increased eNOS-Hsp90 interactions, decreased eNOS-derived superoxide production, and enhanced maximal NOS activity in carnitine-treated shunt lambs were associated with improved NO signaling, as demonstrated by increased plasma $\mathrm{NO}_{\mathrm{x}}$ levels as compared with those of vehicle-treated shunt lambs (Figure 5d).

\section{Pulmonary Vascular Reactivity}

In the current study, we found that the endothelium-dependent vasodilator acetylcholine (Ach) chloride $(1 \mu \mathrm{g} / \mathrm{kg}) \mathrm{did}$ not decrease pulmonary vascular resistance (PVR) in vehicle-treated shunt lambs (Figure 6). However, in carnitinetreated shunt lambs, PVR decreased significantly in response to acetylcholine (Figure 6). The Ach-mediated decrease in PVR was not significantly different between carnitine-treated shunt lambs and age-matched control lambs with normal PBF (Figure 6). By contrast, in response to the endothelium-independent vasodilator inhaled NO (40 ppm), calculated PVR decreased similarly in all groups (Figure 6). Figure 6 shows percentage change. These data are identical when analyzed for absolute change over time (data not shown).

\section{DISCUSSION}

The results of this study show that chronic L-carnitine treatment prevents the disruption of carnitine homeostasis, reduces oxidative stress, and improves pulmonary mitochondrial function, NO signaling, and endothelial function in our lamb model of CHD with increased PBF. Carnitine is present in the organism as FC or as AC (esterified form), which along with carnitine-dependent enzymes and plasma membrane transporters constitute the carnitine system. Adequate carnitine levels, as well as optimal activities of carnitine-dependent enzymes, are needed to allow the carnitine system to function. The main function of L-carnitine is the transport of long-chain fatty acids from the cytosol to the mitochondrial matrix for $\beta$-oxidation and adenosine triphosphate production. However, L-carnitine also plays a key regulatory role in intermediary metabolism by modulating cellular acyl-coenzyme A (CoA)/CoA ratio. This function is mostly dependent on the freely reversible conversion of short-chain acyl-CoA and carnitine to free CoA and acylcarnitine by the intramitochondrial enzyme CrAT. CoA is an obligate cofactor for many enzymes involved in intermediary metabolism. It remains compartmentalized in limited pools within the cell, mainly in the mitochondria, and is normally kept in homeostasis with carnitine. The reversible transfer of acyl groups from CoA to carnitine ensures the vital maintenance of free CoA pools within the mitochondria and prevents the accumulation of poorly metabolized short-chain acyl-CoA compounds, which are exported out of the mitochondria as carnitine esters. Therefore, the carnitine system is crucial for normal mitochondrial function, as the accumulation of acyl groups and the unavailability of 


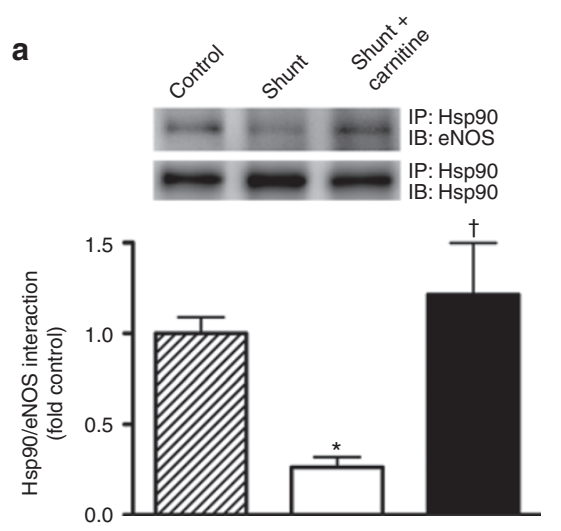

b

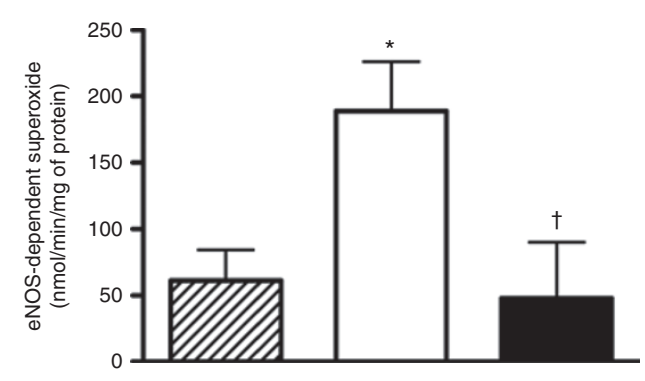

C

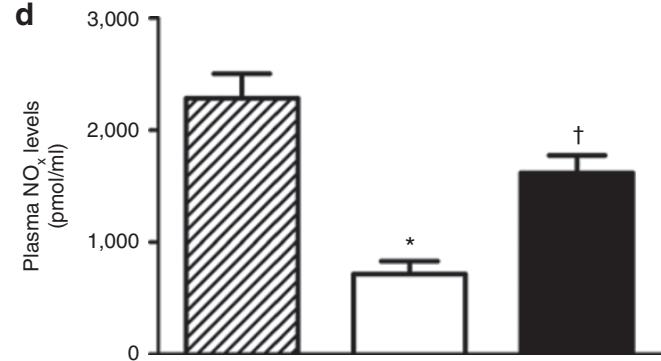

Figure 5. NO signaling in the lamb lung. (a) The interaction of eNOS with $\mathrm{Hsp} 90$ was determined by immunoprecipitation using specific antiserum raised against eNOS in the peripheral lung of vehicle- (white) and carnitine-treated (black) shunt lambs as well as age-matched control lambs (hatched). Immunoprecipitated extracts were analyzed using antisera against either eNOS or Hsp90 (to normalize for immunoprecipitation efficiency). A representative image is shown. (b) Superoxide anion levels were also determined by electronic paramagnetic resonance in snap-frozen lung tissue in each group of lambs, in the presence and absence of the NOS inhibitor, 2-ethyl-2-thiopseudourea $(100 \mu \mathrm{mol} / \mathrm{l})$. (c) Total NOS activity and (d) NO levels were also determined in peripheral lung in all three groups. Values are mean $\pm \mathrm{SEM} ; n=5$ vehicle-treated shunt lambs, $n=6$ carnitine-treated shunt lambs, and $n=6$ age-matched control lambs. ${ }^{*} P<0.05$ vs. control; ${ }^{\dagger} P<0.05$ vs. vehicle-treated shunt lambs. eNOS, endothelial nitric oxide synthase; Hsp, heat shock protein; IB, immunoblot; IP, immunoprecipitation.

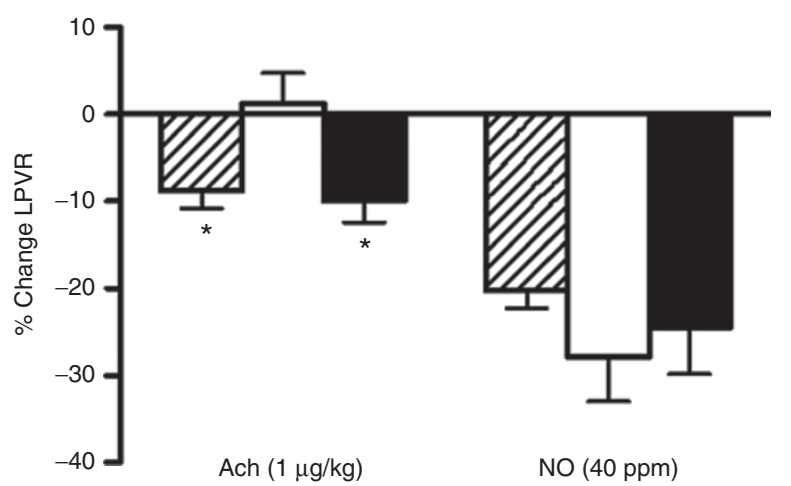

Figure 6. Measurements of left pulmonary vascular resistance (LPVR). Changes in LPVR, expressed as percentage change from baseline, in response to acetylcholine (Ach; $1 \mu \mathrm{g} / \mathrm{kg}$ ), an endothelium-dependent agent, and inhaled nitric oxide (40 ppm), an endothelium-independent agent, in carnitine-treated ( $n=7$, black) or vehicle-treated ( $n=5$, white) shunt lambs and age-matched control lambs ( $n=5$, hatched). Acetylcholine significantly decreased LPVR in the carnitine-treated but not in the vehicle-treated shunt lambs. The decrease in PVR was not significantly different between carnitine-treated shunt lambs and age-matched control lambs. All groups experienced a similar drop in PVR in response to inhaled NO (40 ppm). Values are mean $\pm S D$. ${ }^{*} P<0.05$ compared with baseline.

free CoA result in a metabolic roadblock within the mitochondria, with subsequent impaired oxidative metabolism, increased mitochondrial ROS generation, and decreased energy production (15-17).
We have previously demonstrated disrupted carnitine homeostasis in shunt lambs, and this leads to mitochondrial dysfunction and attenuated NO signaling (14). These lambs showed high $\mathrm{AC} / \mathrm{FC}$ ratios, reflecting an imbalance in mitochondrial acyl-CoA/CoA, as well as a decreased expression of three important carnitine-dependent enzymes (CPT1, CPT2, and CrAT) and a nitration-dependent decrease in CrAT activity. Despite compelling evidence that oxidative stress plays a causal role in the development of pulmonary vascular disease secondary to increased PBF (13), this study was the first to suggest a mitochondrial component linked to alterations in the carnitine system in its pathogenesis. Previously, lung mitochondrial dysfunction had only been reported in the pulmonary hypertension syndrome observed in fast-growing broilers, which was interestingly attenuated by antioxidant therapy with vitamin $\mathrm{E}$ (18). Different mechanisms probably explain this disrupted carnitine homeostasis in our lamb model of increased PBF. It has been proposed that under conditions of metabolic stress such as ischemia-reperfusion injury, the endogenous pool of carnitine can become insufficient for the acyl transfer demand, leading to a carnitine insufficiency state resulting in an increased mitochondrial acyl-CoA/CoA ratio and impaired mitochondrial function $(19,20)$. Other evidence suggests that mitochondrial oxidative stress damages CrAT, decreasing its binding affinity for substrates and resulting in mitochondrial dysfunction and further oxidative stress (21). However, adequate exogenous 


\section{Articles | Sharma et al.}

carnitine supplementation can overcome this oxidative inhibition $(15,19,21)$. Endothelial NOS is uncoupled in shunt lambs (14). Uncoupled eNOS, through mechanisms not fully elucidated, redistributes from the plasma membrane to the mitochondria, where it induces nitrosative stress by increasing the nitration of mitochondrial proteins (22); our prior in vivo data indicate that eNOS-dependent CrAT nitration contributes to the disruption of carnitine homeostasis, which results in mitochondrial dysfunction and subsequent impaired adenosine triphosphate production (14). Previous studies have shown the importance of adenosine triphosphate in pulmonary endothelial function, as demonstrated by its key role in the birth-related pulmonary vasodilation in fetal lambs, a role likely due to its ability to stimulate NO release via the activation of eNOS (23). eNOS activity is tightly controlled through multiple mechanisms that include phosphorylation and protein-protein interactions. Hsp90, a member of a molecular chaperone family, is among the proteins that increase eNOS activity. Therefore, it is plausible that if, as suggested by our data, disruption in carnitine homeostasis decreases Hsp90/eNOS interactions and attenuates NO production (14), L-carnitine supplementation would result in improved endothelial function. It is important to note that as well as eNOS, GTP cyclohydrolase I, the rate-limiting enzyme in tetrahydrobiopterin biosynthesis, is also chaperoned by Hsp90 $(24,25)$. Tetrahydrobiopterin levels are reduced in shunt lambs and also preserved by L-carnitine supplementation $(24,25)$. Therefore, the preservation of NO signaling and endothelial function in L-carnitine-supplemented shunt lambs probably involves increases of GTP cyclohydrolase I / Hsp90 as well as eNOS/hsp90 interactions.

In the current study, we show how chronic L-carnitine supplementation preserved lung carnitine homeostasis in shunt lambs, decreasing the AC:FC ratio. In addition, L-carnitine reduced levels of nitrated CrAT, and this improved the activity of the enzyme. Furthermore, these alterations in the carnitine system were associated with improved mitochondrial function, as demonstrated by a significantly lower lactate/pyruvate ratio and improved NO signaling. Data indicating this improvement include a significant decrease in Hsp70 protein levels, an increase in eNOS-bound Hsp90, and enhanced $\mathrm{NO}_{x}$ levels, as well as reduced eNOS-derived superoxide in carnitine-treated shunt lambs. Of note, all these changes translated functionally into enhanced endothelial function, as demonstrated by a conserved reduction of PVR in response to Ach. The physiological improvement appears to be selective to the endothelium because the response to inhaled NO was unchanged.

Prior studies have evaluated L-carnitine as a therapeutic tool in other conditions characterized by mitochondrial dysfunction and oxidative stress. In addition to reducing the toxicity resulting from excess acyl-CoA, exogenous L-carnitine has been shown to have antioxidant and antiapoptotic properties (26-28). The mechanisms by which $\mathrm{L}$-carnitine protect cells against ROS is not completely clear but may include direct free-radical scavenging and inhibition and/or repair of peroxidized biomolecules $(27,29)$. L-Carnitine supplementation has also been shown to enhance NO production and attenuate oxidative stress and endothelial dysfunction in systemic hypertensive rats $(30,31)$. With respect to pulmonary vascular disease, in a recent study in cold-exposed broilers with pulmonary hypertension syndrome, L-carnitine supplementation showed beneficial effects on lipid peroxidation and pulmonary vascular remodeling and postponed the occurrence of pulmonary hypertension syndrome for $1 \mathrm{wk}$. Nevertheless, it did not reduce cumulative pulmonary hypertension syndrome mortality (32). In addition, propionyl-L-carnitine increased eNOS protein expression in the same animal model (33). Finally, two recent small studies on children with sickle cell disease and $\beta$-thalassemia-associated pulmonary arterial hypertension suggested a benefit of $\mathrm{L}$-carnitine therapy in decreasing pulmonary artery systolic pressure $(34,35)$.

It is also worth noting the potential limitations of our study, especially regarding the oral delivery system we utilized $(100 \mathrm{mg} / \mathrm{kg} / \mathrm{d})$. It has been shown that oral administration of carnitine occurs both by carrier-mediated transport and through passive diffusion. However, this process appears to be relatively inefficient because previous studies using oral doses of $1-6 \mathrm{~g}$ resulted in only $5-18 \%$ bioavailability as compared with the $75 \%$ bioavailability of L-carnitine ingested through dietary means. Therefore, supplemental doses of L-carnitine appear to be absorbed less efficiently (36). The dose for this study was chosen after three pilot studies in shunt lambs demonstrated that free and acylcarnitine lung levels returned to values similar to those of nonoperated controls (20). It is unclear how L-carnitine supplementation led to an increase in the expression of CPT1. However, it is possible that this may be mediated via an increase in the activity of peroxisome proliferator-activated receptor- $\gamma$ (PPAR $\gamma)$. We have previously shown that PPAR $\gamma$ expression and activity are reduced in shunt lambs (37), and studies indicate that the expression of at least some of the carnitine homeostasis genes can be regulated by PPAR (38). This study also demonstrated that the promoter region of the CPT1 gene contains a PPAR response element (38). However, a PPAR response element has not been identified in the CrAT gene, and thus, it remains to be elucidated how L-carnitine preserves CrAT expression in shunt lambs. Alternatively, these genes may be downregulated in response to oxidative stress, which is reduced in the presence of $\mathrm{L}$-carnitine. However, further studies will be required to elucidate these mechanisms. It is also worth noting that L-carnitine supplementation did not preserve all the parameters we measured to those observed in age-matched control lambs with normal PBF. Of note, although maximal NOS activity was enhanced in carnitine supplemented lambs, $\mathrm{NO}$ levels did not increase above those observed in age-matched control lambs with normal PBF. However, as the reduction in PVR in response to Ach was still preserved, this suggests that there is sufficient bioavailable NO produced in carnitine-supplemented shunt lambs to induce smooth muscle cell relaxation. Finally, it is unclear why, and noteworthy that, none of the mitochondrial inborn errors of metabolism associated with carnitine deficiencies have been shown to be associated with the development of pulmonary hypertension. Although it is possible that this correlation has not been investigated or alternatively, because the therapy 
for carnitine homeostasis defects is high-dose L-carnitine, this could prevent the potential development of pulmonary hypertension.

In conclusion, our results indicate that chronic L-carnitine treatment attenuates the alterations in lung carnitine homeostasis previously demonstrated in our lamb model of CHD with increased PBF, reducing associated oxidative stress and improving pulmonary mitochondrial function, NO signaling, and ultimately endothelial function. Chronic L-carnitine therapy may improve and/or attenuate the decline in endothelial function noted in children with these disorders and thus has important clinical implications that warrant further investigation.

\section{METHODS}

\section{Experimental Model}

This procedure has been previously described in detail (18). A total of 24 mixed-breed western neonatal lambs were utilized in our study. These corresponded to 13 lambs with increased PBF subdivided into two experimental groups receiving daily treatment with oral Lcarnitine $(n=7,100 \mathrm{mg} / \mathrm{kg} / \mathrm{d})$ or its vehicle $(n=6)$. Eleven lambs with normal blood flow served as controls. All studies were carried out at $4 \mathrm{wk}$ of age. At the end of the experimental protocol, all lambs were killed with a lethal injection of sodium pentobarbital followed by bilateral thoracotomy as described in the National Institutes of Health Guidelines for the Care and Use of Laboratory Animals. The committees on animal research of the University of California, San Francisco, and Georgia Regents University approved all protocols and procedures.

\section{Hemodynamic Measurements}

Pulmonary and systemic arterial and right and left atrial pressures were measured using Sorenson Neonatal Transducers (Abbott Critical Care Systems, Chicago, IL). Mean pressures were obtained by electrical integration. Heart rates were measured by a cardiotachometer triggered from the phasic systemic arterial pressure pulse wave. Left PBF was measured on an ultrasonic flow meter (Transonic Systems, Ithaca, NY). All hemodynamic variables were measured continuously using the Gould Ponemah Physiology Platform (Version 4.2) and Acquisition Interface (model ACG-16; Gould, Cleveland, OH) and recorded with a Dell Inspiron 5160 computer (Dell, Round Rock, TX). Blood gases and $\mathrm{pH}$ were measured on a Radiometer ABL $5 \mathrm{pH} / \mathrm{blood}$ gas analyzer (Radiometer, Copenhagen, Denmark). Hemoglobin concentration and oxyhemoglobin saturation were measured by a cooximeter (model 682; Instrumentation Laboratory, Lexington, MA). PVR was calculated using standard formulas. Shunt fraction $(\mathrm{Qp} /$ Qs) was determined using the Fick principle. Body temperature was monitored continuously with a rectal temperature probe.

\section{Pulmonary Vascular Reactivity}

Pulmonary vascular responses were then assessed in response to ACh and inhaled NO. Ach chloride $(1 \mu \mathrm{g} / \mathrm{kg})$ followed by inhaled NO (40 $\mathrm{ppm}$ ) was administered. Ach chloride (IOLAB, Claremont, CA) was diluted in sterile $0.9 \%$ saline and delivered by rapid injection into the pulmonary artery. Inhaled NO was delivered to the inspiratory limb of the respiratory circuit (Inovent; Ohmeda, Liberty, NJ) and continued for $15 \mathrm{~min}$. The inspired concentrations of NO and nitrogen dioxide were continuously quantified by electrochemical methodology (Inovent; Ohmeda). The hemodynamic variables were monitored and recorded continuously. A minimum of $30 \mathrm{~min}$ separated the administration of ACh and inhaled NO, and the second agent was not given until baseline hemodynamics returned.

\section{Preparation of Protein Extracts and Western Blot Analysis}

Lung protein extracts were prepared and used for western blot analysis as previously described (3). Briefly, protein extracts $(50 \mu \mathrm{g})$ were separated on Long-Life 4-20\% Tris-sodium dodecyl sulfate-Hepes gels (Gradipore, Frenchs Forest, Australia). All gels were electrophoretically transferred to Immuno-Blot polyvinylidene fluoride membranes (Bio Rad Laboratories, Hercules, CA). The membranes were blocked with 5\% nonfat dry milk in Tris-buffered saline containing $0.1 \%$ Tween-20. After blocking, the membranes were probed at room temperature with antibodies to eNOS (BD Transduction Laboratories, San Jose, CA), CPT1B (Affinity Bioreagents, Rockford, IL), CPT2 (Affinity Bioreagents), CrAT (Santa Cruz Biotechnology, Santa Cruz, CA), Hsp70 (Enzo Life Sciences, Farmingdale, NY), or Hsp90 (BD Transduction Laboratories), washed with Tris-buffered saline containing $0.1 \%$ Tween, and then incubated with an appropriate IgG conjugated to horseradish peroxidase. Protein bands were then visualized with chemiluminescence (SuperSignal West Femto Substrate Kit, Pierce Laboratories, Rockford, IL) on a Kodak 440CF Image Station (Kodak, Rochester, NY). Band intensity was quantified using Kodak 1D image processing software. All captured and analyzed images were determined to be in the dynamic range of the system. To normalize for protein loading, blots were reprobed with the housekeeping protein $\beta$-actin.

\section{Measurement of Carnitine Homeostasis}

For FC (L-carnitine and acetyl-L-carnitine) determination, $100 \mu \mathrm{l} \mathrm{sam-}$ ples, $300 \mu \mathrm{l}$ water, and $100 \mu \mathrm{l}$ of internal standard (Sigma ST 1093) were mixed. For total carnitine determination, $100-\mu \mathrm{l}$ samples were hydrolyzed with $0.3 \mathrm{~mol} / \mathrm{l} \mathrm{KOH}$, heated at $45^{\circ} \mathrm{C}$, and $\mathrm{pH}$-neutralized using perchloric acid; the volume was made to $400 \mu \mathrm{l}$, and $100 \mu \mathrm{l}$ internal standard was added. All samples were purified using solid-phase extraction columns, SAX $100 \mathrm{mg} / \mathrm{ml}$ (Varian, Harbor City, CA) and derivatized using aminoanthracene in the presence of 1-[3-(dimethylamino) propyl]-3-ethylcarbodiimide hydrochloride (EDCI) (catalyst) and kept at $30^{\circ} \mathrm{C}$ for $1 \mathrm{~h}$ to complete reaction of carnitines. Separation was carried out with an isocratic elution in $0.1 \mathrm{~mol} / 1$ Tris-acetate buffer $(\mathrm{pH}$ $3.5)$ : acetonitrile $(68: 32, \mathrm{vol} / \mathrm{vol})$ at a flow rate of $0.9 \mathrm{ml} / \mathrm{min}$ as described $(39,40)$. ACs were calculated as total carnitine minus FC. Detection of carnitines was then performed using high-performance liquid chromatography as we have previously described (14).

\section{Measurement of CrAT Activity}

Peripheral lung tissue was homogenized in $50 \mathrm{mmol} / \mathrm{l}$ Tris- $\mathrm{HCl}(\mathrm{pH}$ 7.5), $2 \mathrm{mmol} / \mathrm{l} \mathrm{EDTA,} 5 \mathrm{mmol} / \mathrm{l} \mathrm{MgCl}_{2}, 0.8 \mathrm{mmol} / \mathrm{l}$ dithiothreitol, and $0.25 \mathrm{mmol} / \mathrm{l}$ phenylmethylsulfonyl fluoride (PMSF) with protease inhibitor cocktail. CrAT activity was then determined as previously described (14).

\section{Immunoprecipitation Analyses}

Peripheral lung tissues were homogenized in immunoprecipitation buffer $(25 \mathrm{mmol} / \mathrm{l} \mathrm{N}$-2-hydroxyethylpiperazine-N9-2-ethanesulfonic acid, pH 7.5, $150 \mathrm{mmol} / \mathrm{l} \mathrm{NaCl}, 1 \%$ Nonidet P-40, $10 \mathrm{mmol} / \mathrm{l} \mathrm{MgCl}_{2}$, $1 \mathrm{mmol} / \mathrm{l} \mathrm{EDTA}$, and $\%$ glycerol supplemented with protease inhibitor cocktail (Pierce Laboratories, Rockford, IL)). Tissue homogenates $(1,000 \mu \mathrm{g}$ of protein) were precipitated either with a rabbit antibody against 3-nitrotyrosine (5 $\mu$; Upstate Biotechnology) or to eNOS (5 $\mu \mathrm{g}$; BD Transduction Laboratories) in $0.5 \mathrm{ml}$ final volume at $4{ }^{\circ} \mathrm{C}$ overnight as previously described (14).

\section{Determination of Lactate and Pyruvate Levels}

This was carried out in peripheral lung tissue as previously described (14).

\section{Assay for NOS Activity}

NOS activity was determined using the conversion of ${ }^{3} \mathrm{H}$-L-arginine to ${ }^{3} \mathrm{H}$-L-citrulline as previously described (3).

\section{Measurement of Superoxide Levels in Peripheral Lung Tissue}

Approximately $0.2 \mathrm{~g}$ of peripheral lung tissue was sectioned from freshfrozen tissue and immediately immersed in either normal EPR buffer (phosphate-buffered saline supplemented with $5 \mu \mathrm{mol} / \mathrm{l}$ diethydithiocarbamate (Sigma-Aldrich, St Louis, MO) and $25 \mu \mathrm{mol} / \mathrm{l}$ desferrioxamine (Sigma-Aldrich)) or EPR buffer supplemented with $100 \mu \mathrm{mol} / \mathrm{l}$ 
3 ethylisothiourea (Sigma-Aldrich), an inhibitor of NO synthases (41). Superoxide levels were then estimated by electronic paramagnetic resonance assay using the spin-trap compound 1-hydroxy-3-methoxycarbonyl-2,2,5,5-tetramethylpyrrolidine. $\mathrm{HCl}$ as we have previously described $(42,43)$. NOS-derived superoxide levels were determined by subtracting the values in the presence of ethylisothiourea from the values in the absence of ethylisothiourea. To convert electronic paramagnetic resonance waveforms into units of superoxide, we used 1 $\mathrm{mU}$ of xanthine oxidase to generate $1 \mathrm{nmol} / \mathrm{l} / \mathrm{min}$ of superoxide over a 60-min period to generate a standard curve. Using this standard curve, we were able to convert waveform amplitudes into nmol of superoxide produced $/ \mathrm{min} / \mathrm{mg}$ protein in each reaction condition.

\section{Measurement of Bioavailable NO ( $\mathrm{NO}_{x}$ )}

Plasma samples were treated with cold ethanol for $1 \mathrm{~h}$ at $-20{ }^{\circ} \mathrm{C}$ and then centrifuged at $20,000 \mathrm{~g}$ to remove proteins that can interfere with NO measurements. Potassium iodide-acetic acid reagent was prepared fresh daily by dissolving $0.05 \mathrm{~g}$ of potassium iodide in $7 \mathrm{ml}$ of acetic acid. KI/AcOH mixture was added into a septum-sealed purge vessel and bubbled with nitrogen gas. The gas stream was connected via a trap containing $1 \mathrm{~N} \mathrm{NaOH}$ to a Sievers 280i Nitric Oxide Analyzer (GE Analytical, Boulder, CO). Samples were injected with a syringe through a silicone-Teflon septum. Results were analyzed by measuring the area under the curve of the chemiluminescence signal using the Liquid software (GE Analytical). The resultant $\mathrm{NO}_{\mathrm{x}}$ value represents total nitric oxide and nitrite.

\section{Statistical Analysis}

Statistical analysis was performed using GraphPad Prism version 5.0 (GraphPad Software, San Diego, CA). The means \pm SD or SEM were calculated. Statistical significance was determined either by the unpaired $t$-test (for 2 groups) or ANOVA (for $\geq 3$ groups) with Tukey's post hoc testing. A value of $P<0.05$ was considered significant.

\section{ACKNOWLEDGMENTS}

The authors thank Sridevi Dasarathy, Johnny Wright, Michael Johengen, and Cynthia Harmon for excellent technical assistance.

\section{STATEMENT OF FINANCIAL SUPPORT}

This research was supported in part by grants HL60190 (to S.M.B.), HL67841 (to S.M.B.), HL084739 (to S.M.B.), R21HD057406 (to S.M.B.), HL61284 (to J.R.F.), and K08 HL086513 (to P.O.), all from the National Institutes of Health, Bethesda, MD; by a grant from the Fondation Leducq (to S.M.B. and J.R.F.); a Scientist Development Grant (11SDG7460024) from the National Affiliates of the American Heart Association, Dallas, TX (to S.S.); and Cardiovascular Discovery Institute Seed Awards from Georgia Regents University, Augusta, GA (to S.S. and S.K.).

Disclosure: The authors declared no conflict of interest.

\section{REFERENCES}

1. Hanley FL, Heinemann MK, Jonas RA, et al. Repair of truncus arteriosus in the neonate. J Thorac Cardiovasc Surg 1993;105:1047-56.

2. Steinhorn RH, Russell JA, Lakshminrusimha S, Gugino SF, Black SM, Fineman JR. Altered endothelium-dependent relaxations in lambs with high pulmonary blood flow and pulmonary hypertension. Am J Physiol Heart Circ Physiol 2001;280:H311-7.

3. Oishi PE, Wiseman DA, Sharma S, et al. Progressive dysfunction of nitric oxide synthase in a lamb model of chronically increased pulmonary blood flow: a role for oxidative stress. Am J Physiol Lung Cell Mol Physiol 2008;295:L756-66.

4. Zhang DX, Gutterman DD. Mitochondrial reactive oxygen speciesmediated signaling in endothelial cells. Am J Physiol Heart Circ Physiol 2007;292:H2023-31.

5. Puddu P, Puddu GM, Cravero E, De Pascalis S, Muscari A. The putative role of mitochondrial dysfunction in hypertension. Clin Exp Hypertens 2007;29:427-34.

6. Genova ML, Pich MM, Bernacchia A, et al. The mitochondrial production of reactive oxygen species in relation to aging and pathology. Ann NY Acad Sci 2004;1011:86-100.
7. Mancuso C, Scapagini G, Currò D, et al. Mitochondrial dysfunction, free radical generation and cellular stress response in neurodegenerative disorders. Front Biosci 2007;12:1107-23.

8. Puddu P, Puddu GM, Cravero E, De Pascalis S, Muscari A. The emerging role of cardiovascular risk factor-induced mitochondrial dysfunction in atherogenesis. J Biomed Sci 2009;16:112.

9. Sims NR, Muyderman H. Mitochondria, oxidative metabolism and cell death in stroke. Biochim Biophys Acta 2010;1802:80-91.

10. Reddy VM, Meyrick B, Wong J, et al. In utero placement of aortopulmonary shunts. A model of postnatal pulmonary hypertension with increased pulmonary blood flow in lambs. Circulation 1995;92:606-13.

11. Reddy VM, Wong J, Liddicoat JR, Johengen M, Chang R, Fineman JR. Altered endothelium-dependent responses in lambs with pulmonary hypertension and increased pulmonary blood flow. Am J Physiol 1996;271(2 Pt 2):H562-70.

12. Grobe AC, Wells SM, Benavidez E, et al. Increased oxidative stress in lambs with increased pulmonary blood flow and pulmonary hypertension: role of NADPH oxidase and endothelial NO synthase. Am J Physiol Lung Cell Mol Physiol 2006;290:L1069-77.

13. Black SM, Fineman JR. Oxidative and nitrosative stress in pediatric pulmonary hypertension: roles of endothelin-1 and nitric oxide. Vascul Pharmacol 2006;45:308-16.

14. Sharma S, Sud N, Wiseman DA, et al. Altered carnitine homeostasis is associated with decreased mitochondrial function and altered nitric oxide signaling in lambs with pulmonary hypertension. Am J Physiol Lung Cell Mol Physiol 2008;294:L46-56.

15. Zammit VA, Ramsay RR, Bonomini M, Arduini A. Carnitine, mitochondrial function and therapy. Adv Drug Deliv Rev 2009;61:1353-62.

16. Pande SV, Blanchaer MC. Reversible inhibition of mitochondrial adenosine diphosphate phosphorylation by long chain acyl coenzyme A esters. J Biol Chem 1971;246:402-11.

17. Ramsay RR, Zammit VA. Carnitine acyltransferases and their influence on CoA pools in health and disease. Mol Aspects Med 2004;25: 475-93.

18. Iqbal M, Cawthon D, Wideman RF Jr, Bottje WG. Lung mitochondrial dysfunction in pulmonary hypertension syndrome. I. Site-specific defects in the electron transport chain. Poult Sci 2001;80:485-95.

19. Wainwright MS, Kohli R, Whitington PF, Chace DH. Carnitine treatment inhibits increases in cerebral carnitine esters and glutamate detected by mass spectrometry after hypoxia-ischemia in newborn rats. Stroke 2006;37:524-30.

20. Wainwright MS, Mannix MK, Brown J, Stumpf DA. L-carnitine reduces brain injury after hypoxia-ischemia in newborn rats. Pediatr Res 2003;54:688-95.

21. Liu J, Killilea DW, Ames BN. Age-associated mitochondrial oxidative decay: improvement of carnitine acetyltransferase substrate-binding affinity and activity in brain by feeding old rats acetyl-L-carnitine and/or R-alpha-lipoic acid. Proc Natl Acad Sci USA 2002;99:1876-81.

22. Sud N, Wells SM, Sharma S, Wiseman DA, Wilham J, Black SM. Asymmetric dimethylarginine inhibits HSP90 activity in pulmonary arterial endothelial cells: role of mitochondrial dysfunction. Am J Physiol, Cell Physiol 2008;294:C1407-18.

23. Konduri GG, Mattei J. Role of oxidative phosphorylation and ATP release in mediating birth-related pulmonary vasodilation in fetal lambs. Am J Physiol Heart Circ Physiol 2002;283:H1600-8.

24. Sharma S, Sun X, Kumar S, et al. Preserving mitochondrial function prevents the proteasomal degradation of GTP cyclohydrolase I. Free Radic Biol Med 2012;53:216-29.

25. Sun X, Fratz S, Sharma S, et al. C-terminus of heat shock protein 70-interacting protein-dependent GTP cyclohydrolase I degradation in lambs with increased pulmonary blood flow. Am J Respir Cell Mol Biol 2011;45: 163-71.

26. Vescovo G, Ravara B, Gobbo V, et al. L-Carnitine: a potential treatment for blocking apoptosis and preventing skeletal muscle myopathy in heart failure. Am J Physiol, Cell Physiol 2002;283:C802-10.

27. Gülçin I. Antioxidant and antiradical activities of L-carnitine. Life Sci 2006;78:803-11. 
28. Calò LA, Pagnin E, Davis PA, et al. Antioxidant effect of L-carnitine and its short chain esters: relevance for the protection from oxidative stress related cardiovascular damage. Int J Cardiol 2006;107:54-60.

29. Arduini A. Carnitine and its acyl esters as secondary antioxidants? Am Heart J 1992;123:1726-7.

30. Herrera MD, Bueno R, De Sotomayor MA, Pérez-Guerrero C, Vázquez CM, Marhuenda E. Endothelium-dependent vasorelaxation induced by L-carnitine in isolated aorta from normotensive and hypertensive rats. J Pharm Pharmacol 2002;54:1423-7.

31. Bueno R, Alvarez de Sotomayor M, Perez-Guerrero C, Gomez-Amores L, Vazquez CM, Herrera MD. L-carnitine and propionyl-L-carnitine improve endothelial dysfunction in spontaneously hypertensive rats: different participation of NO and COX-products. Life Sci 2005;77:2082-97.

32. Tan X, Hu SH, Wang XL. The effect of dietary l-carnitine supplementation on pulmonary hypertension syndrome mortality in broilers exposed to low temperatures. J Anim Physiol Anim Nutr (Berl) 2008;92:203-10.

33. Alvarez de Sotomayor M, Bueno R, Perez-Guerrero C, Herrera MD. Effect of L-carnitine and propionyl-L-carnitine on endothelial function of small mesenteric arteries from SHR. J Vasc Res 2007;44:354-64.

34. El-Beshlawy A, Abd El Raouf E, Mostafa F, et al. Diastolic dysfunction and pulmonary hypertension in sickle cell anemia: is there a role for L-carnitine treatment? Acta Haematol 2006;115:91-6.

35. El-Beshlawy A, Youssry I, El-Saidi S, et al. Pulmonary hypertension in beta-thalassemia major and the role of L-carnitine therapy. Pediatr Hematol Oncol 2008;25:734-43.

36. Evans AM, Fornasini G. Pharmacokinetics of L-carnitine. Clin Pharmacokinet 2003;42:941-67.
37. Tian J, Smith A, Nechtman J, et al. Effect of PPARgamma inhibition on pulmonary endothelial cell gene expression: gene profiling in pulmonary hypertension. Physiol Genomics 2009;40:48-60.

38. Mascaró C, Acosta E, Ortiz JA, Marrero PF, Hegardt FG, Haro D. Control of human muscle-type carnitine palmitoyltransferase I gene transcription by peroxisome proliferator-activated receptor. J Biol Chem 1998;273:85603.

39. Longo A, Bruno G, Curti S, Mancinelli A, Miotto G. Determination of L-carnitine, acetyl-L-carnitine and propionyl-L-carnitine in human plasma by high-performance liquid chromatography after pre-column derivatization with 1-aminoanthracene. J Chromatogr B, Biomed Appl 1996;686:129-39.

40. Minkler PE, Brass EP, Hiatt WR, Ingalls ST, Hoppel CL. Quantification of carnitine, acetylcarnitine, and total carnitine in tissues by high-performance liquid chromatography: the effect of exercise on carnitine homeostasis in man. Anal Biochem 1995;231:315-22.

41. Garvey EP, Oplinger JA, Tanoury GJ, et al. Potent and selective inhibition of human nitric oxide synthases. Inhibition by non-amino acid isothioureas. J Biol Chem 1994;269:26669-76.

42. Wiseman DA, Wells SM, Hubbard M, Welker JE, Black SM. Alterations in zinc homeostasis underlie endothelial cell death induced by oxidative stress from acute exposure to hydrogen peroxide. Am J Physiol Lung Cell Mol Physiol 2007;292:L165-77.

43. Wiseman DA, Wells SM, Wilham J, Hubbard M, Welker JE, Black SM. Endothelial response to stress from exogenous $\mathrm{Zn} 2+$ resembles that of NO-mediated nitrosative stress, and is protected by MT-1 overexpression. Am J Physiol, Cell Physiol 2006;291:C555-68. 